\title{
CARTAS DE PASANTES COMO TEXTOS PEDAGÓGICOS: MOMENTOS DE LA DOCENCIA EN EDUCACIÓN INFANTIL
}

\section{LETTERS FROM INTERNS AS PEDAGOGICAL TEXTS: SNAPSHOTS OF TEACHING IN EARLY CHILDHOOD EDUCATION}

\author{
Rodrigo Saballa de Carvalho \\ Universidade Federal do Rio Gran do Sul, Brasil \\ https://orcid.org/0000-0002-8899-0998 \\ rsaballa@terra.com.br \\ Bianca Salazar Guizzo \\ Universidade Luterana do Brasil,Brasil \\ https://orcid.org/0000-0003-1080-2210 \\ bguizzo_1@hotmail.com
}

Recibido: 22-01-2020 Revisado: 19-02-2020. Aceptado: 01-03-2020

Resumen: El presente artículo, a partir de las contribuciones de los estudios sobre formación docente y de los aportes de los procesos que se desarrollan en pasantías para la Educación Infantil, tiene como objetivo discutir la dimensión pedagógica de las cartas producidas por graduados académicos en Pedagogía. El corpus de análisis consiste en un conjunto de cartas producidas por pasantes (alumnado en práctica) en Educación Infantil. Metodológicamente, a partir de la lectura de las cartas se lleva a cabo un análisis del contenido y la selección fragmentos (los cuales son denominados en este estudio como instantáneas), que muestran la recurrencia del abordaje de algunas temáticas. De esta manera, se definen tres unidades de discusión: a) imagen del niño; b) didáctica docente; c) contexto de escucha de los niños. A partir del análisis, fue posible inferir la importancia de la escritura de cartas como una forma de expresión, reflexión, intercambio de experiencias y, sobre todo, autoformación de docentes durante el período de prácticas.

Palabras claves: Educación Infantil, pasantía, cartas, textos pedagógicos, docencia.

\begin{abstract}
This article, based on the contributions of studies on teacher training, as well as on the processes of internship in Early Childhood Education, aims to discuss the pedagogical dimension of letters produced by academic graduates of a Pedagogy course. The corpus of analysis comprises a set of letters produced by Child Education interns. Methodologically, from the reading of the letters, an analysis is performed of their content and the selection of passages (which are called snapshots) that show the recurrence of the approach of some themes. In this way, three discussion units are defined: a) child image; b) teaching didactics; c) children's listening context. From the analyses, it was possible to infer the importance of letter writing as a way of expression, reflection, sharing experiences and, above all, teaching self-training during the internship period.
\end{abstract}

Keywords: Early Childhood Education, internship, cards, pedagogical texts, teaching.

Cómo citar este artículo: Carvalho, R. S. y Guizzo, B. S (2020). Cartas de pasantes como textos pedagógicos: momentos de la docencia en Educación Infantil. Hachetetepé. Revista científica en Educación y Comunicación, (20), 5-16. doi: http://doi.org/10.25267/Hachetetepe.2020.i20.3 


\section{CONSIDERACIONES INICIALES}

¡Hola! Qué tal, ¿muy ansiosa? ¿Te has dado cuenta de que ha llegado el tan esperado séptimo semestre? Bueno, ahora es solo alegría, de verdad. Podría pasar horas escribiendo todo lo increíble que sucedió este semestre, solo que pasarías toda la mañana leyendo. Por lo tanto, trataré de ser breve. Permítete involucrarte con los niños, con los maestros en el aula, en resumen con todos. Esto ayudará mucho en el desarrollo de la pasantía. Compártelo. Comparta su trabajo de enseñanza con sus colegas, sus amigos, su familia e incluso con sus contactos de redes sociales. Crea conexiones. Confieso que al comienzo de la pasantía me sentí un poco inseguro, pero gradualmente, a medida que buscaba referencias y apoyo del maestro y el grupo de pasantes, vi el potencial que tendría mi pasantía. Dónese a usted mismo. En serio, dónate cada momento para este etapa, porque ahora puede parecer muy distante, pero cuando te des cuenta ya estarás planeando la última semana. Sobre todo... arrase... brille, ¡has que estos niños se vuelvan locos! (P1 (1), transcripción del trecho de la carta, fragmento nuestro).

La formación docente no es un producto, sino un lugar de luchas y conflictos, un contexto fructífero para construir formas de ser y estar en la profesión (Fontana, 2005). La formación, como advierten Ongari y Molina (2005), no termina después de la graduación, sino que continúa desarrollándose en el curso de la vida profesional. Es decir, estamos constituidos como maestros a partir del conjunto de experiencias que tenemos. La maestra que trabaja en Educación Infantil tiene la posibilidad de convertirse en profesional, desenterrando los sentidos en busca de una poética de lo cotidiana, en la que se comprenda tanto el valor educativo del conocimiento (como un proceso de investigación cultural y colaborativo) como la relevancia del proceso dinámico de descubrimiento, creación y experimentación de los niños en la construcción diaria de sus caminos en el mundo. Esto se debe a que, según Cerisara (2002), Kulisz (2004), Fontana (2005), la formación docente está marcada por historias de vida, la formación de las condiciones laborales concretas y los imaginarios recurrentes acerca de la profesión. En esta perspectiva, defendemos la posición de que la identidad de la maestra de Educación Infantil no existe en un sentido fijo e inmutable, sino formas de ser maestra, siempre responsable de problematización, que le permiten construir y reinventar su desempeño profesional siempre que sea necesario. (Alliaud y Antelo, 2009).

En este sentido, cabe destacar que la docencia en Educación Infantil es una actividad humana interactiva, en la que la relación con los niños es el objeto de trabajo y, por lo tanto, un aspecto fundamental en la composición de los modos de ser docentes (Tardif y Lessard, 2014). No es una relación entre la profesora y un objeto inanimado, sino una relación entre seres humanos, capaces de actuar y afectarse mutuamente (Tardif y Lessard, 2014). Defendemos el argumento de que la enseñanza con niños se basa en el encuentro con el otro, lo que hace que la relación sea una condición para su existencia (Alliaud; Antelo, 2009). Desde esta perspectiva, es importante resaltar el movimiento de reconocimiento profesional de la acción educativa de las profesoras en las instituciones de Educación Infantil. Esto se debe a que, la enseñanza en la Educación de la Primera Infancia como una actividad históricamente se relaciona con el trabajo femenino en el hogar, durante mucho tiempo produjo una concepción esencialista y naturalizada de la profesión (Kulisz, 2004). Por otro lado, aunque en las últimas décadas en el Brasil, ha habido una expansión de las inscripciones en Educación Infantil, una profundización de los debates y estudios sobre el niño en la búsqueda de la construcción de una Pedagogía de la Infancia y la integración entre las funciones de cuidar y educar se ha consolidado en 
el plano teórico y legal, sabemos que, en general, la enseñanza con niños de 0 a 5 años de edad, en muchos casos, todavía está estructurada teniendo como referencia la escuela de enseñanza fundamental.

Por lo tanto, contemporáneamente, consideramos que existe la necesidad de inventar otra docencia (Bondioli y Mantovani, 1998) en Educación Infantil, con el objetivo de problematizar las gramáticas pedagógicas (Oliveira-Formosinho, 2003) preceptivo que prohíbe la reflexión sobre los modos de ser docente. Por este motivo, consideramos que es el tiempo/espacio de la pasantía curricular que puede garantizar experiencias y proporcionar un aprendizaje específico, vinculado a la profesión y su propio campo. Por lo tanto, este artículo, basado en las contribuciones de los estudios sobre la formación de docentes (Cerisara, 2002; Silva, 2003; Fontana, 2005; Ongari y Molina, 2005; Alliaud y Antelo, 2009; Tardif y Lessard, 2014), así como al respeto de los procesos de pasantía en Educación Infantil (Ostetto, 2008; Mantovani y Perani, 2009; Drumond, 2019) tiene como objetivo discutir la dimensión pedagógica de las cartas producidas por académicas del curso de Pedagogía.

El corpus de análisis está compuesto por un conjunto de cartas producidas por pasantes (alumnado en prácticas) de Educación Infantil, al momento de completar el Curso de Pedagogía en la Universidad Federal de Rio Grande do Sul (UFRGS). La propuesta de redacción de cartas ocurre regularmente a través de una invitación del profesor orientador (2) al final del período de pasantía. En tal ocasión, las pasantes están invitadas a escribir una carta a sus colegas que realizarán la pasantía en el siguiente semestre. En esta carta, las pasantes deben informar sus desafíos, angustias, experiencias, superaciones y aprendizajes durante la pasantía en Educación Infantil. En este contexto, las cartas llegaron a entenderse como textos pedagógicos (Contreras, 2010), en los que se comparten modos de ejercer la docencia. Metodológicamente, a partir de la lectura de las cartas, realizamos un análisis de su contenido y la selección de extractos (que llamamos instantáneas) que muestran la recurrencia del enfoque de algunos temas en las narraciones escritas por las pasantes.

Así, a través de la lectura de las cartas definimos las siguientes unidades de discusión: a) la construcción de la imagen de un niño virtuoso; b) la invención de la didáctica docente; c) la práctica pedagógica cotidiana de una pedagogía de la escucha. A partir del análisis, fue posible inferir la importancia de la escritura de cartas como una forma de expresión, reflexión, intercambio de experiencias y, sobre todo, autoformación de docentes durante el período de la pasantía. Esto se debe a que, a partir del análisis de las cartas, fue posible hacer visibles los procesos de formación docente de las pasantes, así como las inspiraciones para que podamos avanzar en la construcción de una Pedagogía Infantil, a partir de la construcción de nuevas formas de ejercer la enseñanza con bebés y niños pequeños (Mantovani y Perani, 1999). Justificamos este argumento, según lo declarado por Alliaud y Antelo (2009) cuando afirman que una mejor definición del papel de los maestros de Educación Infantil, diferenciada de la del maestro de Escuela Primaria, solo será posible en la medida en que el trabajo con bebés y niños sea específico, por tanto el trabajo con bebes y niños en la primera infancia debe ser comprendido.

Por lo tanto, el texto está organizado en seis secciones. A partir de la sección introductoria, en la segunda sección, discutimos las especificidades de la pasantía en Educación Infantil como un espacio para la capacitación y la reflexión sobre la práctica docente. En la tercera sección, discutimos las formas en que los alumnos construyen la imagen de un niño virtuoso durante la pasantía. Respectivamente, en la cuarta sección, 
presentamos los modos como la pasantes a partir de presupuestos de la "didáctica del hacer" (Bondioli y Mantovani, 1998) ejercen la práctica docente. En la quinta sección, presentamos la pedagogía de la escucha y su resonancia en la práctica de las pasantes. Finalmente, en la última sección, presentamos las consideraciones finales.

\section{LA PASANTÍA COMO ESPACIO DE FORMACIÓN: NOTAS SOBRE LA DOCENCIA EN EDUCACIÓN INFANTIL}

Los estudios sobre la pasantía docente como un campo de formación deben producir diálogos entre la Educación Infantil y la producción teórica que se ha dedicado a la formación de profesores. Esto se debe a que "la pasantía docente es un espacio privilegiado para la formación del profesorado" (Drumond, 2019, p.2). El propósito de la pasantía es "producir conocimiento y reflexiones sobre la práctica pedagógica, vinculada principalmente al contexto de las instituciones educativas (Drumond, 2019, p.2).

En este sentido, Cerisara (2002) llama la atención sobre la necesidad de profundizar la discusión sobre las características de actuación con niños pequeños, y enfatiza la importancia de construir una nueva mirada a esta actividad. Observar aquello que tiene su comienzo marcado por las prácticas de pasantía en Educación Infantil (Ostetto, 2008; Drumond, 2019). En el desempeño de los profesionales que trabajan con niños muy pequeños, especialmente los bebés, movilizan dimensiones para llevar a cabo una práctica que, tradicionalmente, no ha sido reconocida como profesional. En este sentido, la práctica del docente de pasantía "representa la oportunidad de reflexionar sobre el proceso de formación del profesorado y también la posibilidad de problematizar la vida cotidiana de las guarderías y centros preescolares" (Drumond, 2019, p.2).

Defendemos que "la práctica docente en Educación Infantil gana relevancia en la medida en que esta institución tiene sus propias características y tiene una propuesta educativa diferenciada de la Escuela Primaria" (Drumond, 2019, p.3).

Con el objetivo de identificar las representaciones sociales de los maestros de guardería y preescolar sobre su propio trabajo, Silva (2010) identifica la ausencia de una identidad en la Educación Infancia. El investigador señala que la existencia de dos profesionales diferentes, una maestra de guardería y una maestra de preescolar, que trabajan en el mismo espacio de trabajo, favorece el auto reconocimiento profesional, debido a la forma en que conciben sus prácticas (Silva, 2010). En esta relación, el trabajo que se ocupa del cuidado necesario del niño está más cerca del profesor de guardería, algo desvalorizado, ya que no exige saberes y competencias reconocidas socialmente como siendo profesionales.

A partir de las consideraciones, es posible decir que la constitución del profesional como docente ocurre en un universo marcado por relaciones de diferentes naturalezas: las relaciones adulto - niño en la familia, las relaciones adulto - niño en el contexto institucional, las relaciones entre el profesional del Educación Infantil y las familias de los niños, la relación entre los profesionales de la Educación Infancia y los docentes de otros niveles educativos, así como con el Poder Público (Ongari y Molina, 2005). En este sentido, entendemos que la pasantía ocupa una posición privilegiada en la formación docente, ya que "se entiende como un contexto de formación dialéctica entre teoría y práctica" (Drumond, 2019, p.5).

Además, Alliaud y Antelo (2009) afirman que el proceso de construcción de la identidad docente comienza mucho antes de que el individuo tenga la pretensión de ser un maestro. Para los autores, desde el primer día en que entran en contacto con la 
institución escolar y comienzan a experimentar las prácticas institucionales desarrolladas en ese contexto, inmediatamente comienzan a aprender ejemplos de: a) enseñanza; b) uso del tiempo; c) uso del espacio y d) control de los propios cuerpos, que promueven la interiorización del orden escolar. Se puede decir, entonces, que en el largo camino que los futuros maestros recorren durante todos los años que son estudiantes, "aprenden modelos de enseñanza, conocimiento y reglas de acción, pautas de comportamiento, esquemas sobre la vida, creencias duraderas, imágenes sobre los profesores y su trabajo" (Alliaud y Antelo, 2009), además de los supuestos y valores con respecto a la práctica educativa. En vista de lo anterior, podemos decir que la pasantía también es el momento oportuno para discutir las experiencias que los académicos tuvieron como estudiantes durante sus trayectorias.

Por lo tanto, es necesario problematizar la evidencia en la práctica docente (la forma escolar de educar a bebés y niños pequeños, la disociación entre cuidado y educación, la didáctica de la transmisión, el autoritarismo, el currículo por actividades, las rutinas inflexibles que hacen que los tiempos de la infancia sean inviables, etc.) para que sean visibles durante las pasantías de Educación Infantil procesos de formación docente, basados en la construcción de una imagen de un niño virtuoso (Malaguzzi, 2011; Rinaldi, 2012; Hoyuelos, 2009), en la invención de una "didáctica del hacer" (Bondioli y Mantovani, 1998) y en el ejercicio diario de la escucha (Altimir, 2010).

La definición de la profesora como un profesional adecuado para trabajar con bebés y niños pequeños está en construcción (Mantovani y Perani; 1999) y, por esta razón, debe visibilizarse nuevos procesos de construcción de identidad profesional. En algunos centros de cuidado se realizan prácticas que atribuyen a la profesora el papel de la planificación y el desarrollo de actividades pedagógicas y al profesional auxiliar solamente los cuidados de asistencia física (Silva, 2003; Kulisz, 2004). Además, todavía hay prácticas desarrolladas con niños de 3, 4 y 5 años, preparatorias para la educación primaria, que a menudo constituyen, en situaciones sin relevancia para los niños, que denotan una visión del profesor como el centro del proceso educativo.

\section{EL EJERCICIO ÉTICO DE CONSTRUIR LA IMAGEN DE UN NIÑO}

Colega, enfréntate a la pasantía como un momento para despedirte de aquella buena y vieja forma de ser estudiante y comienza enfrentar la vida profesional como maestra de Educación Infantil, con profesionalismo, ética y respeto hacia los niños. Además, permítete entrar en la emoción de estar con los niños todos los días. Tienen mucho que ofrecer. Por lo tanto, ofrézcales también "mucho", en relación con el afecto, la atención y las poderosas propuestas que proporcionarán. No juzgue a los niños ni los etiquete. Los niños son mega curiosos, grandes investigadores y están dotados de un deseo incomparable de creación. Mejore aún más estas cualidades que tienen, sin haberlas subestimado. Son protagonistas de su propio hacer y deben ser vistos como personas poderosas. ( $\mathrm{P} 4$, transcripción de un trecho de la carta - fragmento nuestro).

El proceso de observación diaria de los niños es fundamental. Ver el potencial de los niños de su grupo será el primer paso para desarrollar una pasantía exitosa. Por lo tanto, invierta en observación en momentos en que los niños desarrollan propuestas en grupos pequeños. Es a través de la observación de los niños que los conocerá y podrá construir una imagen de un niño capaz de investigar, relacionar, expresar opiniones y ser protagonista en el proceso educativo. ( $\mathrm{P} 7$, transcripción de un trecho de la carta fragmento nuestro). 
La lectura de las cartas posibilita evidenciar que las pasantes construirán durante la práctica docente una imagen de un niño virtuoso, que debe respetar sus especificidades generacionales y garantizar su protagonismo. Según Malaguzzi (2011), la educación comienza con la imagen del niño. De esta manera, el autor reitera, la imagen es una convención cultural y una miríada de imágenes de niños que pueden ser elaboradas por los maestros que trabajan con ellos (Hoyuelos, 2009). Por otro lado, cada maestro tiene su propia imagen de un niño, que se refleja indefectiblemente en la expectativa que tiene cuando lo mira (Hoyuelos, 2009).

En este sentido, es fundamental que las pasantes construyan una imagen de un niño basada en referencias teóricas éticas y políticas (Rinaldi, 2012), como defienden las pasantes en sus cartas. Como señala Hoyuelos (2009), la forma en que vemos a los niños es el reflejo del punto de vista que tenemos sobre las personas, ya que la imagen de un niño está directamente relacionada con la vida. Para ello, es necesario invertir en la formación de profesores, que sepan definir sus intercesores teóricos, capaces de producir pedagogía a través de una acción pedagógica consciente a través de la interacción con los niños y el reconocimiento del camino de aprendizaje que siguen. Por lo tanto, desde la etapa de enseñanza, es necesario que el futuro maestro aprenda "a observar a los niños, identificar sus modalidades comunicativas más elementales y establecer una relación comunicativa específica con ellos" (Mantovani; Perani, 1999, p.13).

Como explicó Hoyuelos (2009), los niños y los maestros experimentan procesos paralelos y convergentes en la vida diaria institucional, ya que cada uno de ellos, a partir de sus experiencias, elabora preguntas fundamentales sobre el significado de su vida y existencia. Metafóricamente, es como si el maestro estuviera frente a un espejo, es decir, cuando aprende a ver el potencial de los niños, el maestro también ve su propio potencial de manera correlacionada (Hoyuelos, 2009), como se puede ver en la transcripción de cartas presentadas.

En esta perspectiva, la imagen del niño es un proceso importante en la constitución docente de la pasante, ya que de esta forma se ve al niño "como un sujeto social, un ciudadano que emerge en el escenario social, político y cultural compuesto de vida y no solo de necesidades" (Rinaldi, 1999, p.77). Esta imagen será una declaración de principios éticos para comenzar a trabajar y establecer una didáctica con los niños, ya que puede considerarse como la intersección entre las referencias teóricas de la maestra y su práctica docente. En otras palabras, la imagen de un niño es la base sobre la cual se basa todo el proyecto educativo en la Escuela Infantil (Hoyuelos, 2009).

Por lo tanto, la imagen del niño es un factor esencial en la construcción de la identidad social y ética del maestro de Educación Infantil (Malaguzzi, 2011). Corroborando el argumento, Rinaldi (1999), asegura que la imagen de un niño virtuoso en el contexto de la escuela está directamente relacionada con la identidad de un maestro que también es virtuoso (Rinaldi, 1999, p.78). Por lo tanto, es necesario que el maestro se convierta en el profesional que el niño necesita, ya que ella no es un simple trabajador, pero un intérprete que mejora el encuentro de los niños con la vida (Hoyuelos, 2009).

\section{LA PRÁCTICA DE UNA DIDÁCTICA EN EL EJERCICIO DE LA DOCENCIA CON LOS NIÑOS}

¡La pregunta clave de la pasantía es ensuciarse las manos, sin miedo, sin pereza, sin quejas y con mucha voluntad y energía para hacerlo realidad! Nada cae del cielo. Tienes todas las herramientas necesarias para hacer una hermosa pasantía. Marque la diferencia en la vida de 


\section{Hachetetepé. Revista científica de Educación y Comunicación}

$\mathrm{N}^{\mathrm{o}} 20,6-16,2020$

los niños, ser un buen maestro, respetarlos, darles la bienvenida, jugar con ellos y proponer contextos poderosos, inusuales, provocativos y creativos. Finalmente, la "didáctica de hacer" es un camino fuerte que debe seguir durante la pasantía (P1, transcripción de un trecho de la carta - fragmento nuestro).

La planificación de contextos, teniendo en cuenta tiempos, espacios y materiales es muy importante. Te darás cuenta durante el comienzo de la pasantía que todo lo que escuchaste durante la clase finalmente comenzará a tener sentido. Después de todo, la planificación del contexto debe tener una intención pedagógica. Tal intencionalidad, surge de una didáctica que se construye en la vida cotidiana con los niños. No hay espacio para realizar actividades, trabajar con propuestas que tengan continuidad. Por lo tanto, siempre es necesario pensar en lo que se propone, la forma en que se propone y la intención de la propuesta. Es esencial mantener la continuidad en sus propuestas y en los procesos de investigación de los niños. Este proceso de trabajo es hermoso y muy valioso. ¡Atrévete! Invierte en una "didáctica del hacer", operando en la práctica con las discusiones teóricas desarrolladas en clase (P8, transcripción de un trecho de la carta - fragmento nuestro).

¿Cuál es la didáctica de hacer (Bondioli y Mantovani, 1998) mencionada por las pasantes en sus cartas? Tal didáctica no se refiere a una versión educativa disciplinar y propedéutica cuyo objetivo es desarrollar habilidades para una vida futura, pero es una forma de respetar las características específicas de los niños y garantizarles un espacio para que desarrollen su aprendizaje a partir de contextos en los que puedan hacer ejercicio, curiosidad a través de investigaciones, elaboración de preguntas $y$ planteamiento de hipótesis. Como se puede ver en la lectura de las cartas de las pasantes, la didáctica del hacer (Bondioli y Mantovani, 1998) tensiona la identidad de un maestro de Educación Infantil como transmisor de conocimiento, el cual, basado en un concepto curricular por actividades, define las propuestas consideradas "pedagógicas" en detrimento (Bondioli y Mantovani, 1998) de las demandas de los niños. Es una didáctica en la que se reconoce la importancia de lo inusual, un enfoque en el que las profesoras se ven obligadas a pensar en la planificación de tiempos, espacios y materiales, creando contextos significativos para que los niños vivan sus experiencias de aprendizaje en la vida diaria de la escuela (Augusto, 2015).

$\mathrm{El}$ aspecto fundamental de la didáctica del hacer (Bondioli y Mantovani, 1998) es la construcción de una imagen de un niño potente (Rinaldi, 2012); es decir, el enfoque está en el reconocimiento del niño como sujeto de derechos. Según Rinaldi (1999, p.114), en esta perspectiva se defiende "que el niño tiene potencial, plasticidad, curiosidad, capacidad para maravillarse y el deseo de relacionarse con otras personas y comunicarse". El desafío para el maestro "es aprender a estar presente sin ser un intruso, apoyar conflictos productivos desafiando las respuestas de los niños e involucrarse para revivir una situación, cuando los niños están perdiendo interés" (Rinaldi, 2012, p.117). Esto se debe a que "uno nunca debe olvidar la importancia del papel de la profesora al ofrecer un entorno, materiales y equipos estimulantes para los niños" (Rinaldi, 2012, p.117).

Para conocer esta forma de aprendizaje, Bondioli y Mantovani (1998) indican la necesidad de construir otro modo de ejercitar la docencia, es decir, una maestra que no da clases (Russo, 2007). Además, tales autores, como Fochi (2015), destacan la alegría, la continuidad y la importancia de las experiencias como principios de la didáctica del hacer. En este caso, de acuerdo con la imagen que el maestro tiene de los niños, se convierte en sus propios objetivos educativos (Hoyuelos, 2009). La autonomía, la socialización, la capacidad de construir el mundo al explorarlo, se convierten en objetivos 
educativos para ser respetados y garantizados. En esa dirección, según Fochi (2015), organizar campos de experiencias en vista del juego, la continuidad y la importancia de lo que experimentan los niños en las escuelas es también una forma de resaltar las concepciones de la educación escolar, de los niños, de los profesores.

A partir de este punto de vista, Fochi (2015, p. 225) comprende lo lúdico "como una forma de favorecer al niño en el ejercicio creativo, desde un espacio y tiempo para formular teorías". Sin embargo, según Bondioli y Mantovani (1998, p.32) "el juego no es un criterio suficiente para mantener el proceso de crecimiento en relación con la construcción del mundo exterior". Esto se debe a que las experiencias de aprendizaje de los niños implican tiempo, un hecho que corrobora la necesidad de definir la continuidad como un segundo principio de la didáctica del hacer (Fochi, 2015). A medida que los niños experimentan una realidad basada en un tiempo no cronológico, es la continuidad de sus caminos de investigación lo que garantiza la posibilidad de que los niños establezcan relaciones entre sus investigaciones, descubrimientos y los innumerables problemas planteados durante el proceso de construcción del conocimiento. La continuidad, conforme Fochi (2015, p.226) implica la planificación de tiempos, materiales, espacios y la formación de pequeños grupos. Esto se debe a que "es en la continuidad de las experiencias que la fuerza y la vitalidad de la acción de los niños reside en comprender, explorar y profundizar sus hipótesis afectivas, cognitivas y sociales sobre el mundo" (Fochi, 2015, p.226). Finalmente, la didáctica del hacer implica el principio de importancia que se compone de "autoría, elección y provisionalidad de los significados construidos por los niños" (Fochi, 2015, p.227). Es decir, la forma en que los niños significan su aprendizaje también se hace visible a través de la didáctica del hacer.

De este modo, a partir de las consideraciones presentadas, es posible inferir que la didáctica del hacer (Bondioli y Mantovani, 1988), a través de sus principios lúdicos, de continuidad y de significación, se configuran como un proceso importante en la constitución de la docencia de la profesora de Educación. Infantil. Esto se debe a que, desde una didáctica que subvierte las prácticas de transmisión, la maestra tiene la posibilidad de entenderse a sí misma como maestra involucrada en la construcción de una nueva forma de enseñar.

\section{LA PRÁCTICA COTIDIANA DE LA ESCUCHA EN EL ENCUENTRO CON LOS NIÑOS Y SUS PARES}

Escuchar a los niños es una tarea compleja al comienzo de la pasantía. Compleja, porque no es suficiente con escuchar, debe hacer posible que las ideas, sugerencias y posicionamientos de los niños entren en la planificación y se conviertan en parte de la vida diaria que todos ustedes experimentan. Es importante recordar que escuchar no solo ocurre en el momento del círculo (como tradicionalmente ocurre en las instituciones). La escucha se produce en todo momento en la mañana, desde el momento en que recibe a los niños, hasta su partida. Ponerse en la posición de aquellos que escuchan a los niños es una actitud ética que debe desarrollarse durante la pasantía. Estoy seguro de que enfrentará muy bien este desafío ( $\mathrm{P} 8$, transcripción de un trecho de la carta - fragmento nuestro).

Escuchar es una forma ética de comprender para comprender la cultura infantil, es decir, sus formas de pensar, hacer, preguntar y teorizar (Hoyuelos, 2009). Escuchar significa estar atento a los niños en sus relaciones con el mundo, como lo destacan las pasantes en sus cartas. La idea de escuchar es parte de la ética educativa que constituye 
la enseñanza en Educación Infantil (Hoyuelos, 2009), ya que permite el establecimiento de relaciones más igualitarias entre niños y adultos. Como recuerda Ostetto (2008, p.129), "la necesidad de mirar al niño, observar a los diferentes niños con los que trabaja el maestro, se ha convertido en un principio educativo". Este argumento se reitera en los escritos de las cartas transcritas, cuando ambos pasantes enfatizan la importancia de escuchar en sus prácticas de enseñanza. El niño pequeño solicita a la profesora una pedagogía sostenida en la escucha, cuyas prácticas educativas se centran intencionalmente en sus experiencias diarias y sus procesos de aprendizaje en el contexto de la vida colectiva (Hoyuelos, 2009; Altimir, 2010). Por lo tanto, es necesario invertir en la construcción de una escuela infantil que se centre en el niño y, como opción pedagógica, la oferta de una poderosa experiencia infantil en la que se discuta y comparta socialmente la calidad.

Escuchar es una actitud que presupone disponibilidad para interpretar los mensajes compartidos en el colectivo. Es una actitud que la maestra puede adoptar si cree en un modelo educativo participativo, en el cual los niños tienen la oportunidad de crear y construir significados (Altimir, 2010). Ahora, escuchar a los niños es decisivo para que comprendan el significado, el placer y el valor de comunicar (Altimir, 2010). Por las razones explicadas, consideramos que escuchar es una habilidad importante en la formación del maestro de Educación Infantil. Del mismo modo, tampoco es posible entender lo que los niños quieren (Hoyuelos, 2009). Por esta razón, defendemos que en la Educación Infantil el tiempo privilegiado debe ser estar junto con los niños.

\section{CONSIDERACIONES FINALES}

La imagen del niño (Rinaldi, 2012; Hoyuelos, 2009), la didáctica del hacer (Bondioli y Mantovani, 1998; Fochi, 2015) y la práctica de la escucha (Altimir, 2010), se presentaron durante el transcurso del artículo como aspectos conceptuales recurrentes en cartas producidas por las pasantes, que muestran las formas en que operan con las referencias teóricas con las que tuvieron contacto durante la graduación en sus prácticas docentes. En este sentido, entendemos que el proceso de desarrollo personal y profesional de un maestro en formación no ocurre de forma aislada, sino en contextos intensamente relacionales (Alliaud y Antelo, 2009). La pasante está inmersa en su ambiente de trabajo en compañía de niños, en primer lugar, sus colegas profesoras, del equipo de gestoras, del profesor orientador etc. En esta interacción, está en juego su propia historia de vida, que está vinculada a la historia de vida de su grupo de trabajo y su grupo de niños. Ahora, "la experiencia de escuchar y relacionarse con los niños en el transcurso de un año, el conocimiento de la comunidad, su conocimiento académico, los intercambios con los colegas" (Augusto, 2015, p.117) constituyen a las pasantes como profesoras de Educación Infantil. De este modo, los procesos de constitución de la docencia están en sintonía con la figura de maestras que busca continuamente una acción educativa que respete los derechos de los niños y sus familias (Malaguzzi, 2011). Con esto vale la pena decir que, aunque la trayectoria histórica de construir la identidad del maestro de Educación Infantil ha sido marcada por el desprestigio, por la asociación directa con las actividades desarrolladas en el ámbito privado de la casa con respecto al cuidado de los niños y la falta de cualificación profesional (Kulisz, 2004), ha llegado el momento de potenciar los procesos de construcción de la identidad docente que está en línea con una Pedagogía de la Infancia y con el conocimiento que se ha producido y acumulado en las 
últimas décadas, a través de investigaciones que se centran en el niño como actor social competente.

Actualmente con las Directrices Curriculares Nacionales de Educación (Brasil, 2009), es imprescindible que se avance en la discusión sobre los procesos de cualificación de la formación inicial da la profesora de Educación Infantil. Para eso, es necesario invertir en la viabilidad de una formación que permita a los académicos reflexionar sobre las formas en que se están convirtiendo en maestros durante la graduación, en las formas en que son profesoras durante la pasantía y principalmente en las posibilidades que tienen para tomar decisiones en la búsqueda de la invención de una docencia que rompa con la tradición escolarizada y escolarizante de la Educación Infantil. Por tanto, es en ese contexto que defendemos la escritura de cartas como una forma de expresión, reflexión, intercambio de experiencias y, sobre todo, autoformación de docentes (Contreras, 2010) durante el período de prácticas. Este fue el desafío propuesto para las pasantes, para producir a través de la escritura de cartas, reflexiones que interrogan, prueban, confirman, crean, aprecian, marcan y transforman sus modos de ejercer la docencia. Después de todo, no existe un modelo de profesora de Educación Infantil, sino una miríada de posibilidades para la invención pautadas en elecciones éticas, políticas y pedagógicas.

Notas

(1) Para mantener el anonimato de la identidad de los alumnos practicantes (Pasantes), utilizaremos la letra $\mathrm{P}$ para nombrar a los alumnos seguidos de un número que corresponda al orden que hemos definido para las letras. Además, aclaramos que el uso de cartas como material de análisis fue debidamente aceptado por todas las pasantes que participaron en nuestra investigación.

(2) Aclaramos que, aunque el artículo es en coautoría, las cartas se escribieron dentro del alcance del Seminario sobre Educación Infantil, que se lleva a cabo en paralelo con la práctica de pasantía, enseñada y guiada por uno de los autores del texto.

\section{REFERENCIAS BIBLIOGRÁFICAS}

Alliaud, A. y Antelo, E. (2008). Los gajes del oficio:ensenãnza, pedagogía y formación. Buenos Aires: Aique Grupo Editor.

Altimir, D. (2010). Cómo escuchar a la infancia? Barcelona: Octaedro.

Augusto, S. O. (2015). A experiência de aprender na Educação Infantil.EnM.L. Flores y S.S. Albuquerque (Coords). Implementação do Proinfância no Rio Grande do Sul: perspectivas politicas e pedagógicas (pp. 111-118). Porto Alegre: EDIPUCRS.

Bondioli, A. y Mantovani, S. (1998). Manual de Educação Infantil de 0 a 3 anos: uma abordagem reflexiva. Porto Alegre: ArtMed.

Brasil. CNE. Resolução CEB no 01, de 07 de abril de 1999. Define as Diretrizes Curriculares Nacionais para a Educação Infantil. Diário Oficial da União, Brasília, DF, 13 abr. Seção 1, p. 18.

Cerisara, A. B. (2002). Professoras de Educação Infantil: entre o feminino e o profissional. São Paulo: Cortez.

Contreras, J. (2010). Pedagogías de la experiencia y la experiencia de la pedagogía. En J. Contrerasy N. P., Lara (Coords.). Investigar la experiencia educativa (pp. 241271). Madrid: Morata.

Drumond, V. (2019). Estágio e docência na Educação Infantil: questões teóricas e práticas. Olhar de Professor, 22, 1-13.

e-ISSN:2172-7910

Doi: http://doi.org/10.25267/Hachetetepe.2020.i20.3

Universidad de Cádiz 
Fochi, P. S. (2015). Ludicidade, continuidade e significatividade nos campos de experiência. In: Finco, D. et al (Coord.). Campos de experiências na escola da infância: contribuições italianas para inventar um currículo de Educação Infantil brasileiro (pp. 221-232). Campinas: SP: Edições Leitura Crítica.

Fontana, A. y Cação, R. (2005). Como nos tornamos professoras? Belo Horizonte: Autêntica.

Hoyuelos, A. (2009). La ética en el pensamiento y obra pedagógica de Loris Malaguzzi. Barcelona: Octaedro.

Kulisz, B. (2004). Professoras em cena: q que faz a diferença. Porto Alegre: Mediação.

Malaguzzi, L. (2011). La educación infantil en Reggio Emilia. Barcelona: Octaedro.

Mantovani, S. y Perani, R. (1999). Uma profissão a ser inventada: o educador da primeira infância. Pro-Posições, 10(1), 75-98.

Oliveira-Formosinho, J. (2007). Pedagogia(s) da infância: reconstruindo uma práxis de participação. En J. Oliveira-Formosinho, T. M. Kishimoto y M. A., Pinazza (Coords). Pedagogia(s) da Infância: dialogando com o passado, construindo o futuro (pp. 13-36). Porto Alegre: Artmed.

Ongari, B. y Molina, P. (2005). A educadora de creche: construindo suas identidades. São Paulo: Cortez.

Ostetto, L. E. (2008). O estágio curricular no processo de tornar-se professor. En Ostetto, L. E. (Coord.). Educação Infantil: saberes e fazeres da formação de professores (pp. 127-138). Campinas, SP: Papirus.

Rinaldi, C. (1999). O currículo emergente e o construtivismo social. En Edwards, Carolyn; Gandini, Lella; Forman, George. As Cem Linguagens da Criança: a abordagem de Reggio Emilia na educação da primeira infancia (pp. 113-122). Porto Alegre: Artmed.

Rinaldi, C. (2008). Reggio Emilia: a imagem da criança e o ambiente em que ela vive como princípio fundamental. En Gandini, L y Edwards, C. (Orgs).Bambini: a abordagem italiana à Educação Infantil (pp. 75-80). Porto Alegre: Artmed.

Rinaldi, C. (2012). Diálogos com Reggio Emilia: escutar, investigar e aprender. São Paulo: Paz e Terra.

Russo, D. (2007). De como ser professor sem dar aulas na escola da infância. En Faria, A. L. (Coord.). O coletivo infantil em creches e pré-escolas (pp. 67-93). São Paulo: Cortez.

Silva, I. O. (2003). Profissionais da Educação Infantil:formação e construção de identidades. São Paulo: Cortez.

Tardif, M. y Lessard, C. (2014). O trabalho docente: elementos para uma teoria da docência como profissão de interações humanas. Petrópolis: Vozes. 